\title{
Preparation of Ag/HBP/PAN Nanofiber Web and Its Antimicrobial and Filtration Property
}

\author{
Li-Rong Yao, ${ }^{1,2}$ Xiao-Mo Song, ${ }^{3}$ Guang-Yu Zhang, ${ }^{2}$ Shan-Qing Xu, ${ }^{2}$ Ye-Qun Jiang, \\ De-Hong Cheng, ${ }^{1}$ and Yan-Hua Lu${ }^{1}$ \\ ${ }^{1}$ Liaoning Provincial Key Laboratory of Functional Textile Materials, Eastern Liaoning University, Dandong 118001, China \\ ${ }^{2}$ School of Textile and Clothing, Nantong University, Nantong 226019, China \\ ${ }^{3}$ Department of Chemical Environment and Life, Dalian University of Technology, Dalian 116024, China
}

Correspondence should be addressed to De-Hong Cheng; chengdehongldxy@qq.com and Yan-Hua Lu; yanhualu@aliyun.com

Received 27 December 2015; Revised 5 February 2016; Accepted 8 February 2016

Academic Editor: Tamer Uyar

Copyright (C) 2016 Li-Rong Yao et al. This is an open access article distributed under the Creative Commons Attribution License, which permits unrestricted use, distribution, and reproduction in any medium, provided the original work is properly cited.

\begin{abstract}
To widen the application of nanofibers web in the field of medical health materials, a new Ag/amino-terminated hyperbranched polymer (HBP)/polyacrylonitrile (PAN) nanofiber web with excellent antimicrobial activity and filtration property was produced with Ag/HBP dispersion solution and PAN nanofiber. Ag/HBP dispersion solution was prepared with HBP as reducer and stabilizer, and Ag/HBP/PAN nanofiber was prepared by modifying electrospun PAN nanofiber with Ag/HBP aqueous solution. The characterization results showed that spherical Ag nanoparticles were prepared and they had a narrow distribution in HBP aqueous solution. The results of Ag/HBP/PAN nanofiber characterized with SEM and EDS showed that the content of silver nanoparticles on the surface of PAN nanofiber was on the increase when the treating temperature rose. The bacterial reduction rates of HBP-treated PAN nanofiber against $S$. aureus and $E$. coli were about $89 \%$, while those of the Ag/HBP/PAN nanofiber against $S$. aureus and $E$. coli were $99.9 \%$ and $99.96 \%$, respectively, due to the cooperative effects from the amino groups in HBP and Ag nanoparticles. Moreover, the small pores and high porosity in Ag/HBP/PAN nanofiber web resulted in high filtration efficiency (99.9\%) for removing smaller particles $(0.1 \mu \mathrm{m} \sim 0.7 \mu \mathrm{m})$, which was much higher than that of the gauze mask.
\end{abstract}

\section{Introduction}

Nanofibers, which have small pores, a great surface area, and high porosity, are widely used in filtration, Li-ion batteries, wound dressing, and other fields [1-5]. Electrospinning is a useful one-step and straightforward process for fabrication of nanofibers: one is the treatment of multiple working fluids synchronously on one spinneret to create nanofibers with complicated structures such as core-shell or multiple layers $[6,7]$, and the other is after treatment or transition of the electrospun nanofibers for functional applications [8].

Polyacrylonitrile (PAN) is a kind of polymer material with good stability, mechanical properties, and abundant functional cyanogroups $(-\mathrm{CN})$ on its macromolecular chain. The preparation of PAN nanofibers by electrospinning has attracted wide attention, including preparation conditions of PAN nanofibers [9], PAN nanocomposite, and so forth
[10-12]. The nanofiber or nanocomposite web was the useful selective layer for removing smaller particles. Compared to the microfiltration (MF), ultrafiltration (UF), and reverse osmosis (RO) membranes have porosities from 5 to $35 \%$; the nanofiber membranes could easily be made into MF and UF membranes with porosities in range of 80 to $90 \%$ [13]. In addition, the interconnectivity of the pores eliminates the inefficiency of the dead-end pores that are commonly formed in conventional membranes. These characteristics would significantly increase flux and reduce transmembrane pressure drop.

Recently, polymer nanofibers loaded metal nanoparticles have attracted wide interest. Compared with other metals, silver has a unique catalytic performance, high conductivity, and antibacterial, so, more attention has been received. In recent years, electrospun PAN nanofibers loaded silver nanoparticles have been prepared using different methods. 
For example, by the silver mirror reaction (SMR) method, the silver nanoparticles were uniformly dispersed on the surface of PAN nanofibers [14]. And some researchers prepared the silver nanoparticles by electrospinning mixed solution of $\mathrm{AgNO}_{3}$ and PAN, using chemical reduction method to reduce $\mathrm{Ag}^{+}$into $\mathrm{Ag}^{0}$ [15-18]. Using the ion exchange method to get silver nanoparticles from $\mathrm{Ag}_{2} \mathrm{CO}_{3}$, we finally got PAN composite nanofibers [19]. Atmospheric plasma treatment and high temperature were some other methods to prepare electrospun PAN nanofibers loaded silver nanoparticles $[20,21]$. These PAN nanofibers loaded silver nanoparticles have excellent antibacterial and conductive property [2225]. However, the silver nanoparticles prepared in the above research had lower stability because there were no protectors outside them, and the weak combination between silver nanoparticles and PAN nanofiber was another shortcoming.

Hyperbranched polymers (HBP) are a subfamily of dendritic polymers and can be used as effective polymers due to their unique sphere structure network, low viscosity, and functional terminal groups, such as hydroxyl, amine, epoxide, anhydride, carboxylic, and isocyanate groups, especially for amino-terminal HBP which has shown excellent adsorption to several organic polymers and metal-binding behavior [26]. $\mathrm{HBP}$ has an excellent dispersion and stabilization in aqueous, which can be used as a reducer and stabilizer for preparing nanoparticles [27].

In our work, HBP was synthesized and used to prepare silver nanoparticles aqueous solution, and a new Ag/HBP/PAN nanofiber web with excellent antibacterial and filtration property was successfully prepared. At first, the silver nanoparticles with a mean size of about $10 \mathrm{~nm}$ were prepared by mixing $\mathrm{AgNO}_{3}$ and $\mathrm{HBP}$ solution under stirring; the nanosilver solution was used to treat PAN nanofiber web; the concentration of nanosilver solution and the treating temperature were mainly investigated, which were the key factors affecting the content of Ag nanoparticles on PAN nanofibers. Then, the antibacterial activity and filtration properties were also investigated.

\section{Experimental Section}

2.1. Materials. Polyacrylonitrile (PAN, white powder; $\mathrm{Mw}$ $=85,000)$, silver nitrate $\left(\mathrm{AgNO}_{3}\right), \mathrm{N}, \mathrm{N}$-dimethylformamide (DMF), ethylenediamine, methyl acrylate, methanol, and cotton gauze were purchased from Sinopharm Chemical Reagent Co., Ltd. (China) and were of analytical grade. In all the experiments, the chemicals were of analytical grade and all the reagents were used as received without any further purification.

2.2. Preparation of $A g / H B P$ Solution. Hyperbranched polymer (HBP) was synthesized and Ag/HBP solution was prepared according to our previous work in patent [28]. Firstly, ethylenediamine $(30.5 \mathrm{~g}, 0.5 \mathrm{~mol})$ was added to a $500 \mathrm{~mL}$ three-necked round-bottomed glass flask equipped with nitrogen gas protection and magnetic stirring, and methyl acrylate $(43.05 \mathrm{~g}, 0.5 \mathrm{~mol})$ in methanol $(100 \mathrm{~mL})$ was added dropwise to the flask with a magnetic agitator and cooled with an ice bath. Then, the mixture was removed from the ice bath and left for stirring for further $6 \mathrm{~h}$ at room temperature. The mixture was transferred into an eggplantshaped flask for automatic rotary vacuum evaporation, and the temperature was raised to $150^{\circ} \mathrm{C}$ in oil bath for $4 \mathrm{~h}$ under low pressure; after removing the methanol the yellow viscid HBP was obtained.

Then, $0.01 \mathrm{~mol} / \mathrm{L} \mathrm{AgNO}_{3}(20 \mathrm{~mL})$ solution was added dropwise to $2 \mathrm{~g} / \mathrm{L} \mathrm{HBP}(100 \mathrm{~mL})$ solution with constant stirring at $50^{\circ} \mathrm{C}$ for $60 \mathrm{~min}$, and light yellow semitransparent Ag/HBP hybrid was prepared.

2.3. Characterization of $A g / H B P$. Transmission electron microscopy (TEM) images were obtained using an FEI Company (USA) Tecnai G220 transmission electron microscope operating at $300 \mathrm{kV}$ accelerating voltage. Samples were prepared by evaporating the solution of nanoparticles onto a 200-mesh copper grid, which was coated with a carbon support film. The Zetasizer Nano ZS system (90Plus Zeta) from Brookhaven Company (USA) was used to test the size distribution of Ag/HBP. UV-vis spectra from 200 to $900 \mathrm{~nm}$ were obtained using a UV/Visible Absorption Spectrophotometry.

2.4. Preparation of Ag/HBP/PAN Antibacterial Nanofiber Web. The concentration of $12 \mathrm{wt} . \%$ PAN solution was prepared in solvent DMF, and the electrospinning was carried out by applying high voltage between the polymer solution contained in the syringe and the metallic collector; the syringe in this experiment can move from right to left. For all of the electrospinning experiments in this study, aluminum foil acceptor was placed on the collector for the deposition of fibers. The voltage used was $15 \mathrm{kV}$, the feed rate was $0.4 \mathrm{~mL} / \mathrm{h}$, and the needle collector distance was $15 \mathrm{~cm}$. The thickness of nanofiber web was controlled by changing the spinning time.

The PAN nanofiber web prepared as mentioned above was immersed in the $\mathrm{Ag} / \mathrm{HBP}$ solution at $30^{\circ} \mathrm{C}, 60^{\circ} \mathrm{C}$, and $90^{\circ} \mathrm{C}$ in a water bath for $120 \mathrm{~min}$, respectively; the liquor-tofabric ratio was $50: 1$. Another sample was immersed in HBP solution at the same liquor-to-fabric ratio as a control sample. The treated samples were air-dried at room temperature for subsequent characterization.

2.5. Characterization of Ag/HBP/PAN Nanofiber Web. A Hitachi scanning electron microscope (model S-4800, LaB6 gun, Kevex X-ray EDS) was used to observe the morphology of the Ag nanoparticles on the surface of the samples. The diameter of PAN nanofiber was measured by image processing software (Image-Pro Plus 5.0); the BrunauerEmmett-Teller (BET) surface area was measured at $77 \mathrm{~K}$ using a nitrogen adsorption-desorption Micromeritics ASAP-2020 analyzer (Micromeritics Co., USA). The porosity of PAN nanofiber web was calculated by the following equation:

$$
\varepsilon=\left(1-\frac{m}{Z \times A \times \rho}\right) \times 100 \%,
$$

where $\varepsilon$ is the porosity of the relevant fibrous membrane; $m$, $Z$, and $A$ are the mass, mean thickness, and area of per unit 


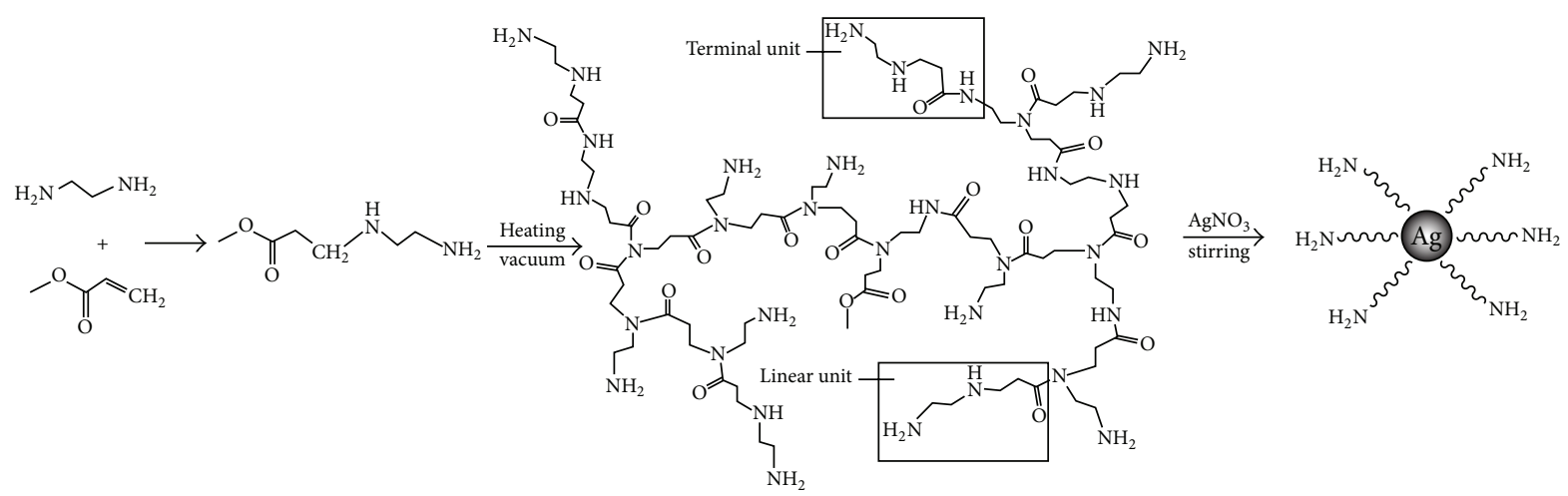

Figure 1: Scheme process of preparing Ag/HBP nanoparticle.

measured membrane, respectively. $\rho$ is the density of polymer raw material and the density of PAN is $1.184 \mathrm{~g} / \mathrm{cm}^{3}$. The nanofiber web was immersed in liquid nitrogen for several minutes and then cut off under liquid nitrogen. The thickness of nanofiber web was measured under optical microscope (Olympus CX31, Japan); the mean thickness was calculated after measurements for 5 times at different cross section.

\subsection{Antimicrobial Activity of Ag/HBP/PAN Nanofiber Web.} The antimicrobial activity of Ag/HBP/PAN nanofiber web was tested against $E$. coli and $S$. aureus by using a shake-flask method according to the literature [29]. The antimicrobial activity of Ag/HBP/PAN nanofiber web was tested against $E$. coli and $S$. aureus by using a shake-flask method according to FZ/T 73023-2006 (China). This method is especially designed for specimens treated with nonreleasing antibacterial agents under dynamic contact conditions. A sample of fabric $(0.5 \mathrm{~g})$, cut into small pieces with size of around $0.5 \times 0.5 \mathrm{~cm}^{2}$, was dipped into a flask containing $70 \mathrm{~mL} 0.3 \mathrm{mM}$ phosphatebuffered saline (PBS, monopotassium phosphate, $\mathrm{pH}$ 7.2) culture solution with cell concentration of $1 \times 10^{5}-4 \times 10^{5}$ colony-forming units (CFU)/mL. The flask was then shaken at $150 \mathrm{rpm}$ on a rotary shaker at $24^{\circ} \mathrm{C}$ for $18 \mathrm{~h}$. From each incubated sample, $1 \mathrm{~mL}$ solution was taken, diluted, and distributed onto an agar plate. All plates were incubated at $37^{\circ} \mathrm{C}$ for $24 \mathrm{~h}$, and the colonies formed were counted. The percentage reduction was determined as follows:

$$
\text { Reduction in CFU (\%) }=\frac{C-C^{\prime}}{C} \times 100 \text {, }
$$

where $C$ and $C^{\prime}$ are the bacterial colonies of the PAN nanofiber web and the Ag-treated PAN nanofiber web, respectively.

2.7. Filtration Property of Ag/HBP/PAN Nanofiber Web. The $\mathrm{Ag} / \mathrm{HBP} / \mathrm{PAN}$ nanofiber web was placed between two layers of cotton gauze to form a sandwich structure; then the filtration of them and cotton gauze with 14 layers were tested. The aerosol filtration efficiency and airflow resistance were measured with a Filter Media Test System (AFC-131, TOPAS Corp., Germany). $\mathrm{NaCl}$ aerosol particles were prepared by an aerosol particle generator (SAG-410, TOPAS Corp., Germany). The neutralized $\mathrm{NaCl}$ aerosol particles were fed into a filter holder and down through the filter with an effective area of $100 \mathrm{~cm}^{2}$. The diameter of sample is $11.5 \mathrm{~cm}$; the test was conducted at room temperature with the continuous air flow rate at the surface of sample fixed at $5.34 \mathrm{~cm} / \mathrm{s}$.

\section{Results and Discussion}

3.1. Preparation of $A g / H B P$. The schematic illustration for fabrication of $\mathrm{Ag} / \mathrm{HBP}$ aqueous solution was given in Figure 1. Hyperbranched polymer (HBP) was synthesized from ethylenediamine and methyl acrylate by melt polycondensation under vacuum. The Ag/HBP aqueous solution was synthesized by mixing $\mathrm{AgNO}_{3}$ aqueous solution and $\mathrm{HBP}$ aqueous solution under vigorous stirring at $50^{\circ} \mathrm{C}$. Abundant amino groups (primary amine, secondary amine, and quaternary amine) inside or at the terminal of HBP macromolecule have reduction performance to reduce $\mathrm{Ag}^{+}$ to silver nanoparticles, and $\mathrm{NH}_{2}$ groups outside the silver nanoparticles play roles of both reductant and stabilizer, which can protect nanosilver particles from oxidation and provide higher security during use [30].

3.2. Characterization of $A g / H B P$. The micromorphology and size distribution of the $\mathrm{Ag}$ nanoparticles are showed in Figure 2. The image of TEM shows that the diameter of $\mathrm{Ag}$ nanoparticles is about $10 \mathrm{~nm}$ and the nanosilvers show a standardized spherical particle and are well dispersed (Figure 2(a)), and the dates from Figure 2(b) further confirm that the Ag nanoparticles prepared have a narrow particle size distribution and the size is about $7 \mathrm{~nm} \sim 13 \mathrm{~nm}$. Results of UVvis adsorption spectra in Figure 2(c) prove the characteristic adsorption peak of $\mathrm{Ag} / \mathrm{HBP}$ at $407.09 \mathrm{~nm}$, confirming the nanocrystalline character of Ag nanoparticles.

3.3. Preparation of $A g / H B P / P A N$ Nanofiber. The process of preparing Ag/HBP/PAN nanofiber web is present in Figure 3. As shown in Figure 3, three steps are needed for preparing Ag/HBP/PAN nanofiber. Firstly, the concentration of 12 wt. $\%$ PAN solution was used for electrospinning and uniform PAN 


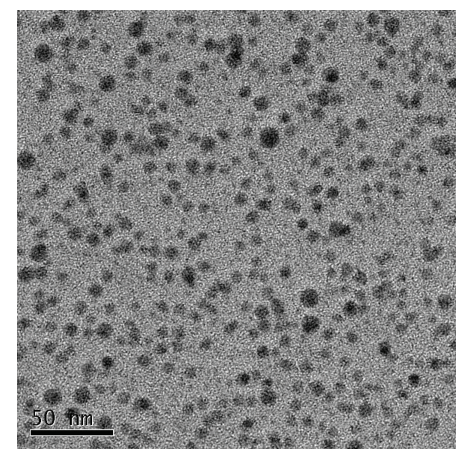

(a) TEM image of Ag/HBP

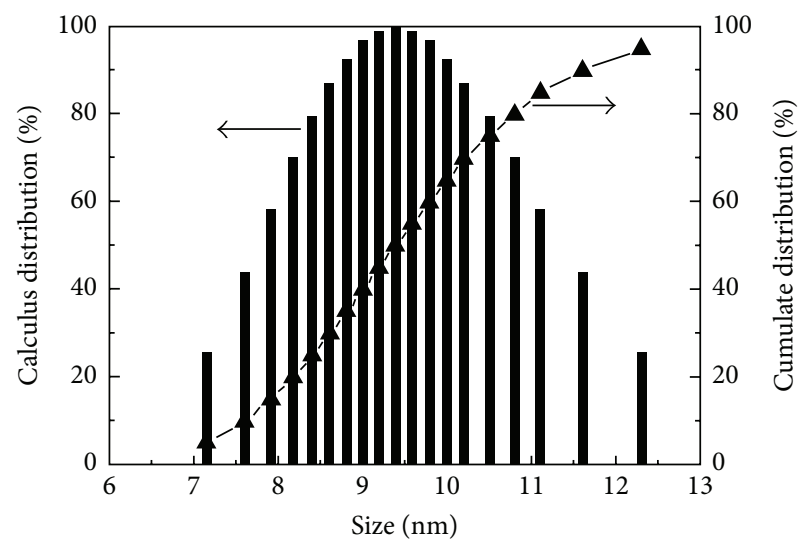

(b) Size distribution of Ag/HBP aqueous

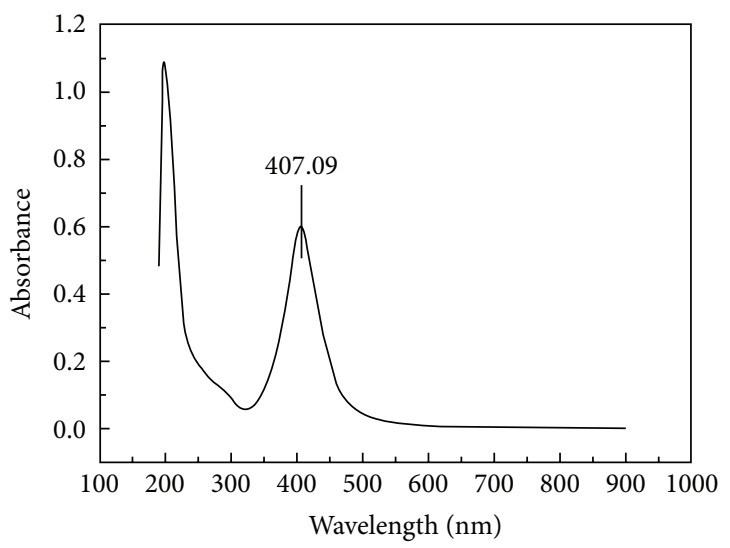

(c) UV-vis adsorption spectra of $\mathrm{Ag} / \mathrm{HBP}$ aqueous

FIGURE 2: Morphology and size distribution of Ag/HBP.

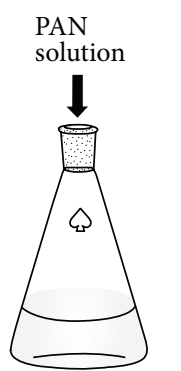

$\stackrel{\text { Electrospinning }}{\longrightarrow}$

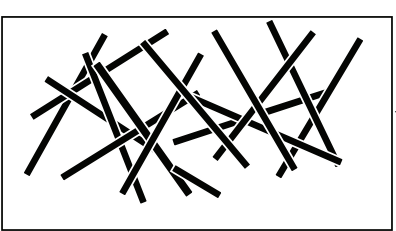

PAN nanofiber web

$\mathrm{HBP}-\mathrm{NH}_{2}$ solution
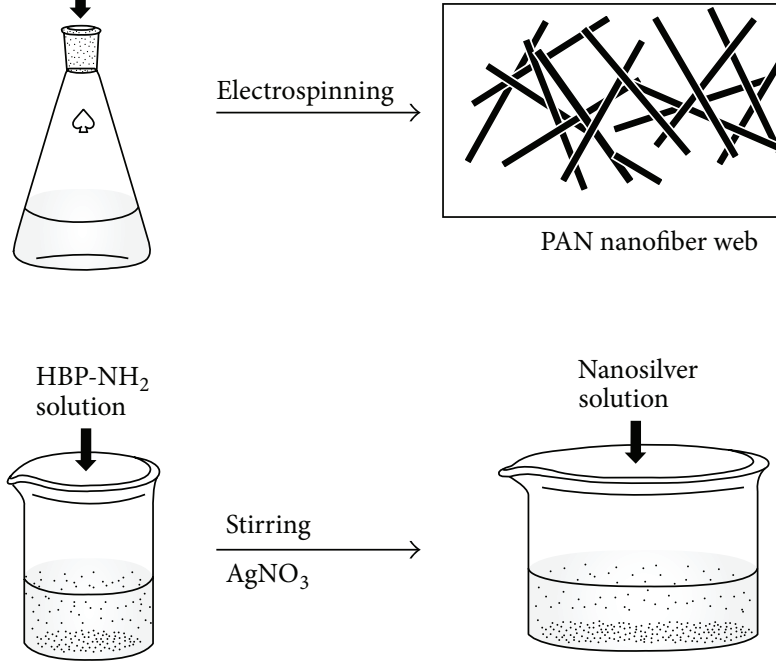

FIGURE 3: Scheme process of preparing Ag/HBP/PAN nanofiber.

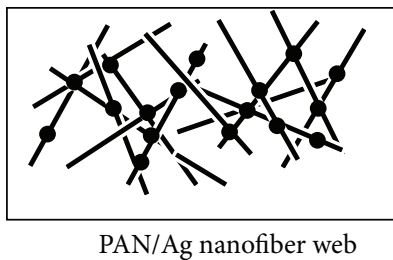

I Nanofiber

- Silver particle 


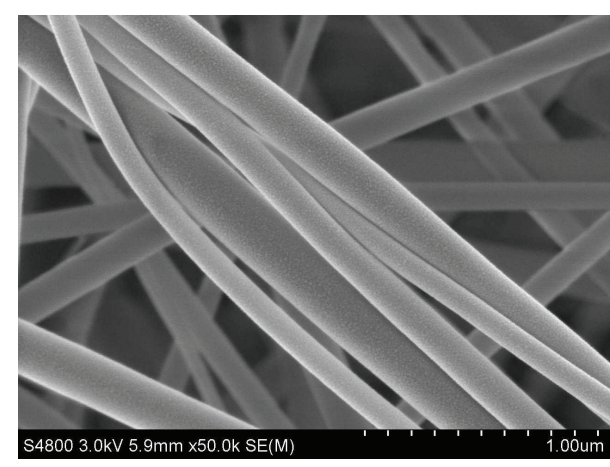

(a) PAN nanofiber

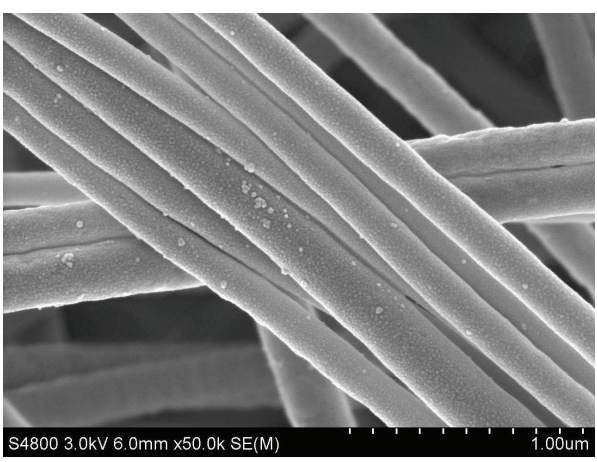

(c) $\mathrm{Ag} / \mathrm{HBP} / \mathrm{PAN}$ nanofiber, 0.05 wt.\%

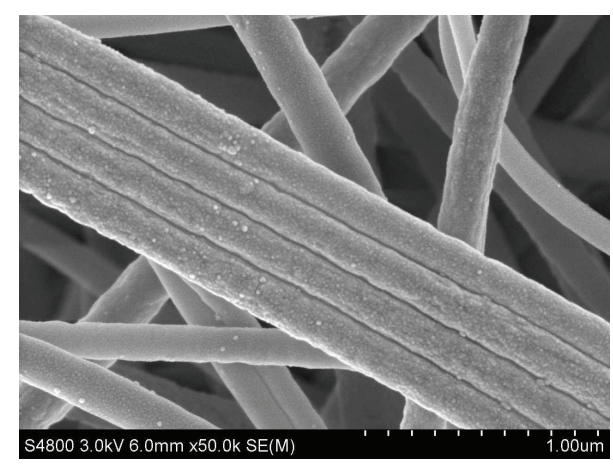

(b) $\mathrm{Ag} / \mathrm{HBP} / \mathrm{PAN}$ nanofiber, $0.02 \mathrm{wt} . \%$

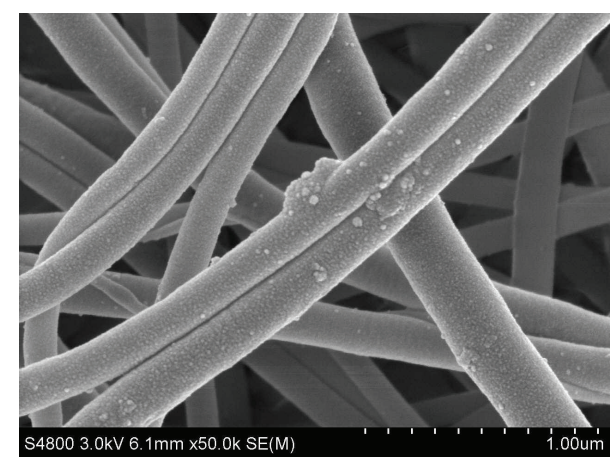

(d) Ag/HBP/PAN nanofiber, 0.08 wt.\%

Figure 4: The morphology of Ag/HBP/PAN nanofiber (temperature of water bath is $30^{\circ} \mathrm{C}$ ).

nanofiber with mean diameter of $200 \mathrm{~nm}-300 \mathrm{~nm}$ was prepared. The second step is to synthesize $\mathrm{Ag} / \mathrm{HBP}$ solution by mixing $\mathrm{HBP}$ and $\mathrm{AgNO}_{3}$ under $50^{\circ} \mathrm{C}$ water bath. Then PAN nanofiber web prepared as mentioned above is immersed in the $\mathrm{Ag} / \mathrm{HBP}$ solution; the concentration of $\mathrm{Ag} / \mathrm{HBP}$ solution and treating temperature are investigated as the main factors affecting the fabrication of $\mathrm{Ag} / \mathrm{HBP} / \mathrm{PAN}$ nanofiber web.

3.4. Characterization of Ag/HBP/PAN Nanofiber. Figure 4 shows the morphology of PAN and Ag-treated PAN nanofibers. As shown in Figure 4, the low treating temperature $\left(30^{\circ} \mathrm{C}\right)$ is used, and no significant increase of silver nanoparticles on the surface of PAN nanofibers is observed in SEM images [Figures 4(b), 4(c), and 4(d)] when the concentration of Ag/HBP solution increased from $0.02 \mathrm{wt} . \%$ to $0.08 \mathrm{wt} . \%$, which indicated that treating temperature plays a much more important role to fix the silver nanoparticles onto the surface of PAN nanofiber.

The SEM pictures of PAN nanofibers loaded silver nanoparticles are shown in Figure 5. From images of Figure 5, no obvious changes are observed on morphology, fiber diameter, and pore size of PAN nanofiber web before and after treatment with $\mathrm{Ag} / \mathrm{HBP}$ solution. However, the silver nanoparticles on the surface of PAN nanofiber have a significant increase when the processing temperature rose from $30^{\circ} \mathrm{C}$ to $90^{\circ} \mathrm{C}$. This indicates that the assembly procedure was temperature dependent. It is believed that the increased electrostatic interaction was responsible for the improved assembly ability.
Specifically, with increasing the temperature, the amino groups $\left(-\mathrm{NH}_{2}\right)$ capped on surface of the silver nanoparticles are highly cationized, endowing the nanoparticle surface with much higher positive charges. Thus the electrostatic interaction between negatively charged PAN nanofiber and positively charged Ag nanoparticles was strengthened. Besides, by the increase of temperature, the functional cyanogroup ($\mathrm{CN})$ on PAN nanofiber and the amino group $\left(-\mathrm{NH}_{2}\right)$ outside the silver nanoparticles have higher activity to combine with each other, and the silver nanoparticles will permeate through the pores inside nanofiber web which have more opportunity to contact with the cyanogroups on PAN nanofiber.

The schematic diagram of hydrogen bonds formation between cyanogroup $(-\mathrm{CN})$ and amino group $\left(-\mathrm{NH}_{2}\right)$ is shown in Scheme 1. Compared to the silver nanoparticles in situ reduced on the surface of fiber, this kind of hydrogen bonding provides stronger combining power between silver nanoparticles and PAN nanofibers. In general, there are two ways to prepare Ag-containing antibacterial materials; this means that the $\mathrm{Ag}$ particles are placed inside of the fiber or fixed on the surface of fiber. For the disposable hygiene products or breathing mask, the Ag particles on surface of fiber show rapid and efficient antimicrobial activity.

3.5. EDS Analysis. EDS test is used to characterize element change on the surface of PAN nanofiber and prove the reaction between $\mathrm{Ag} / \mathrm{HBP}$ solution and PAN nanofibers indirectly, as shown in Figure 6. The results show that carbon, 


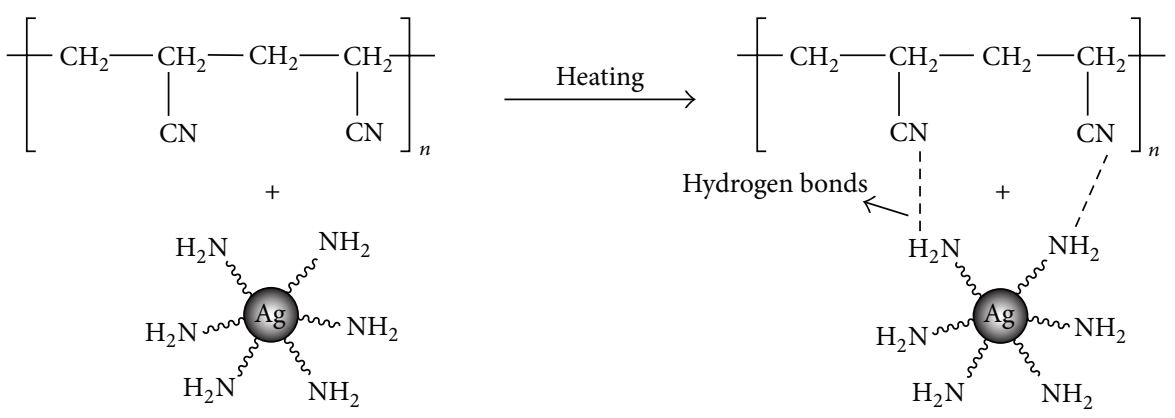

SCHEME 1: Schematic diagram of bonding formation.

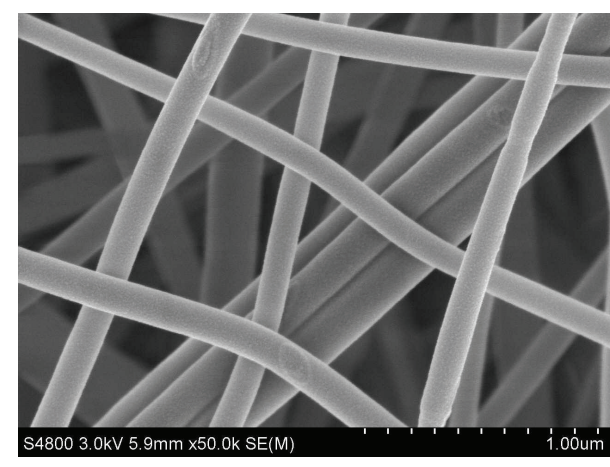

(a) Untreated

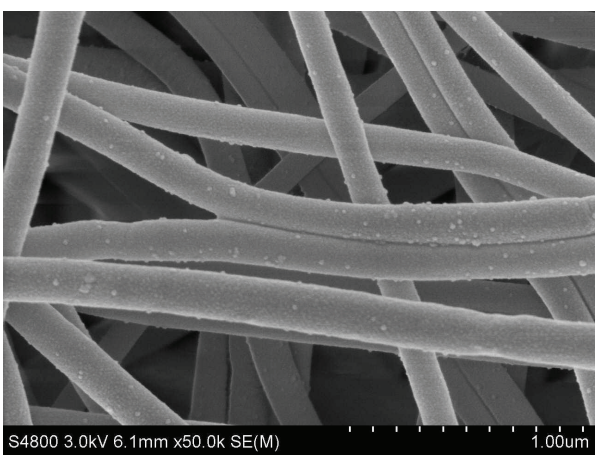

(c) Treated under $60^{\circ} \mathrm{C}$

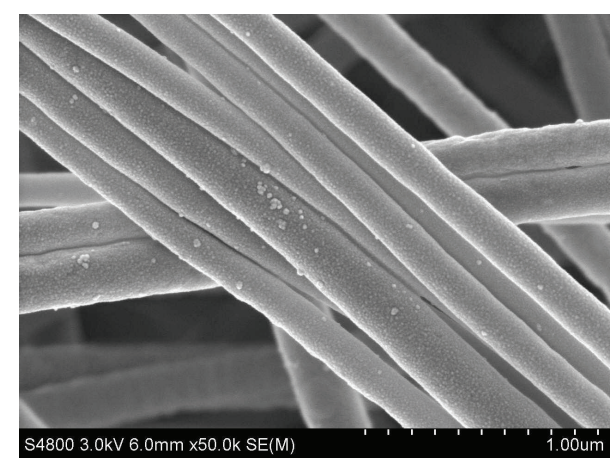

(b) Treated under $30^{\circ} \mathrm{C}$

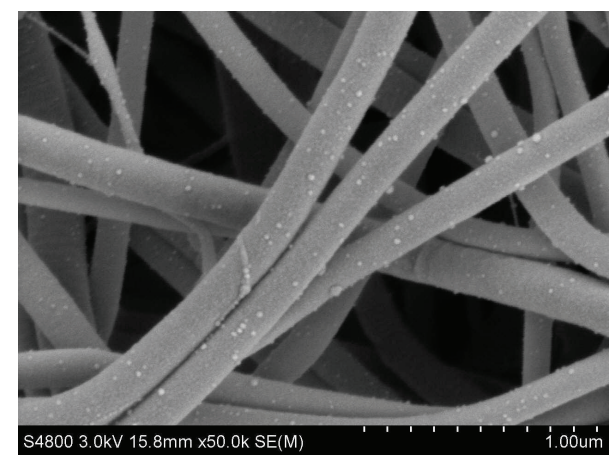

(d) Treated under $90^{\circ} \mathrm{C}$

FiguRE 5: The morphology of Ag/HBP/PAN nanofiber (concentration of silver solution is 0.05 wt.\%).

nitrogen, oxygen, and silver are the principle elements in Ag/HBP/PAN nanofiber web. The EDS analysis provides direct evidence that the silver nanoparticles are fixed on PAN nanofiber surface, which is also identified by SEM images in Figure 5. As shown in Figure 6, the silver content on PAN nanofiber was $2.45 \mathrm{wt} . \%, 8.41 \mathrm{wt} . \%$, and $10.49 \mathrm{wt} . \%$, respectively, when the treating temperature increased from $30^{\circ} \mathrm{C}$ to $90^{\circ} \mathrm{C}$. The increase of Ag content on PAN nanofiber plays an important role in improving its antimicrobial activity.

3.6. Antimicrobial Activity. The antimicrobial activity of the $\mathrm{Ag} / \mathrm{HBP} / \mathrm{PAN}$ nanofiber web is evaluated using $S$. aureus and E. coli and with cotton gauze as control sample. The antimicrobial activity of PAN nanofiber, HBP-treated nanofiber, and
$\mathrm{Ag} / \mathrm{HBP}$-treated nanofiber $\left(0.05 \mathrm{wt} . \%, 90^{\circ} \mathrm{C}\right)$ is presented in Table 1.

Almost the same surviving cells shown in Table 1 indicate that PAN nanofiber shows few actions against $S$. aureus or $E$. coli. The PAN nanofiber web treated with HBP aqueous solution shows antibacterial activity and the bacterial reduction rates against $S$. aureus and E. coli are about $89 \%$. The reason may be ascribed to the abundant amino groups on the PAN nanofiber, which tend to deteriorate the cell membranes of bacteria and induce leakage of intracellular components from bacterial cells, thereby inhibiting their effective growth [29]. After treatment with silver nanoparticles, the Ag/HBP/PAN nanofiber web exhibits excellent antibacterial activity and the bacterial reduction rates against $S$. aureus and E. coli both reached $99.9 \%$, which may be due to the cooperative 


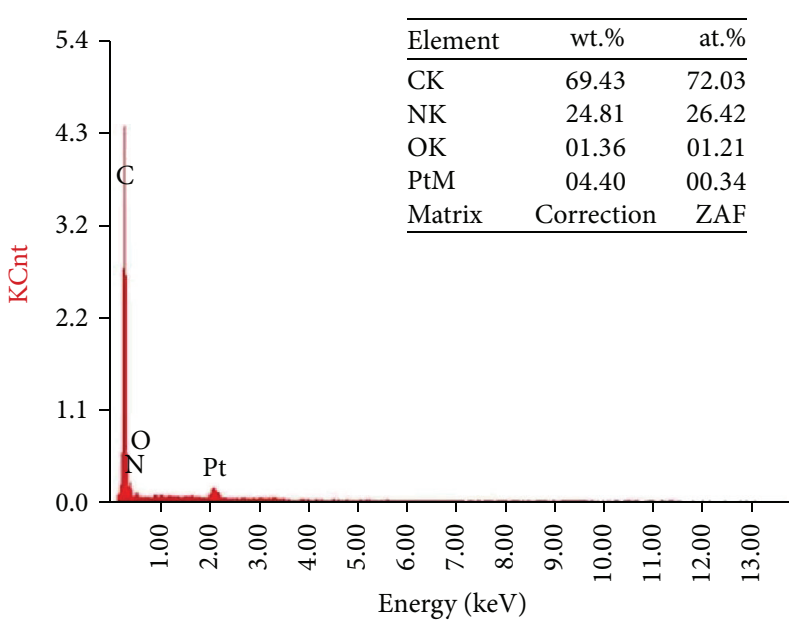

(a) Untreated

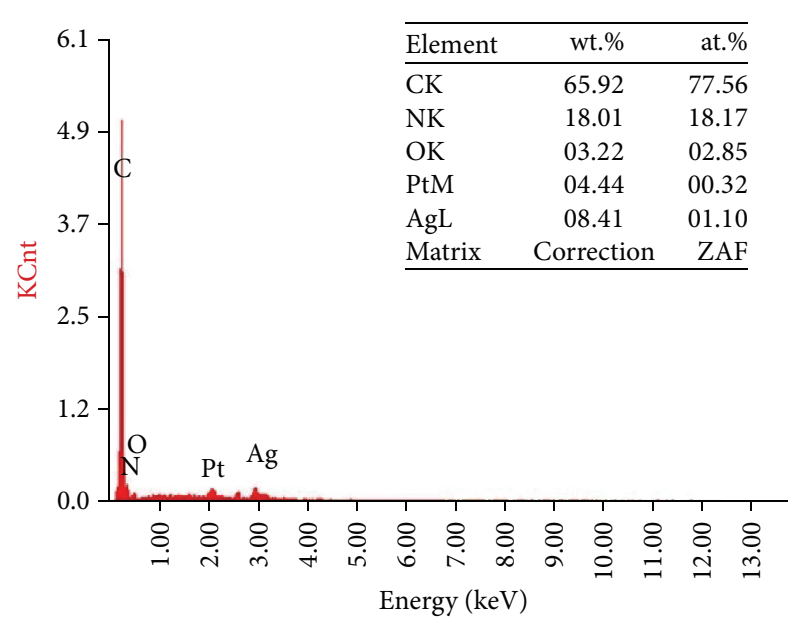

(c) Treated under $60^{\circ} \mathrm{C}$

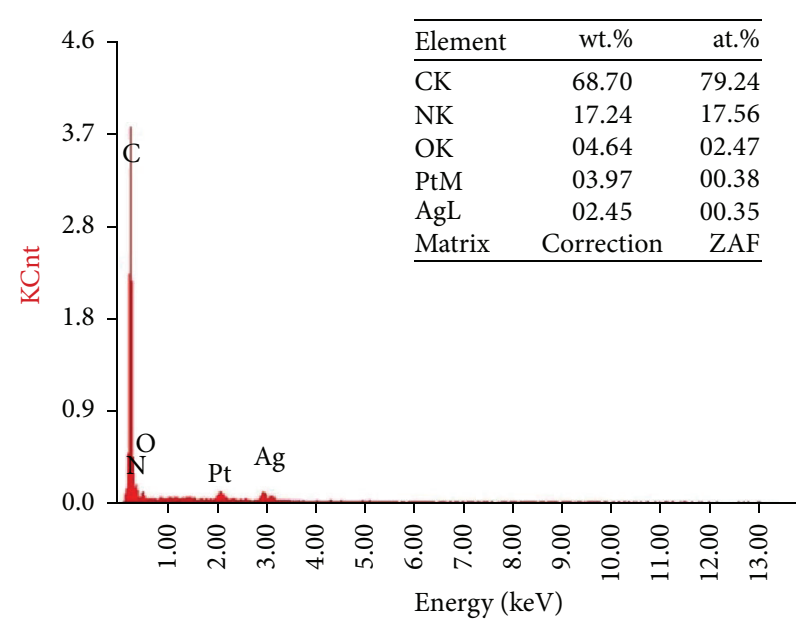

(b) Treated under $30^{\circ} \mathrm{C}$

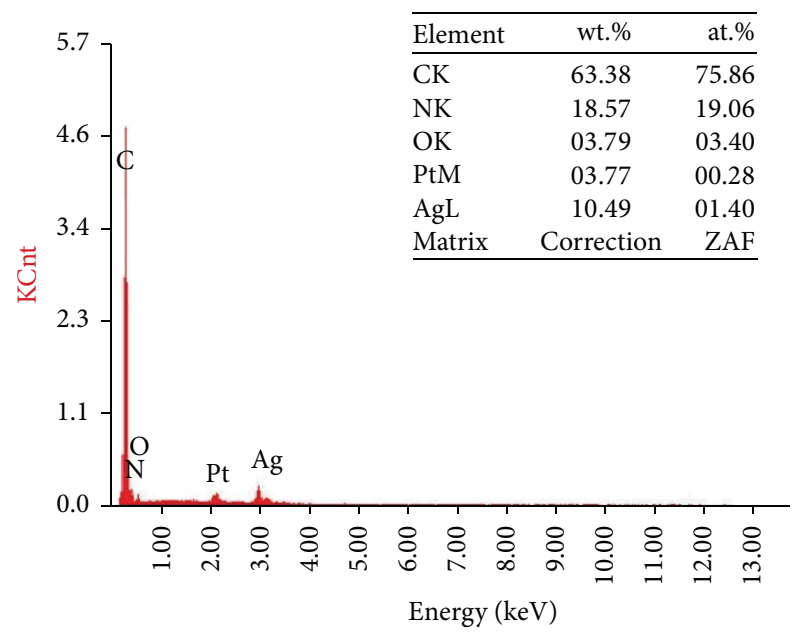

(d) Treated under $90^{\circ} \mathrm{C}$

FIGURE 6: EDS of Ag/HBP/PAN nanofibers (concentration of silver solution is $0.05 \mathrm{wt} . \%$ ).

TABLE 1: Antimicrobial activity of Ag/HBP/PAN nanofiber web.

\begin{tabular}{|c|c|c|c|c|}
\hline \multirow{3}{*}{ Samples } & \multicolumn{4}{|c|}{ Antimicrobial activity } \\
\hline & \multicolumn{2}{|c|}{ S. aureus } & \multicolumn{2}{|c|}{ E. coli } \\
\hline & Surviving cells $(\mathrm{CFU} / \mathrm{mL})$ & $\%$ reduction & Surviving cells $(\mathrm{CFU} / \mathrm{mL})$ & $\%$ reduction \\
\hline Cotton gauze & $1.61 \times 10^{6}$ & & $6.56 \times 10^{6}$ & \\
\hline PAN nanofiber & $1.54 \times 10^{6}$ & 0 & $6.32 \times 10^{6}$ & 0 \\
\hline HBP-treated nanofiber & $1.76 \times 10^{5}$ & 88.57 & $6.71 \times 10^{5}$ & 89.38 \\
\hline Ag/HBP-treated nanofiber & $1.48 \times 10^{3}$ & 99.90 & $2.33 \times 10^{3}$ & 99.96 \\
\hline
\end{tabular}

antimicrobial activity from the amino groups in HBP and Ag nanoparticles on PAN nanofibers.

3.7. Filtration Property. Figure 7 shows the filtration efficiency of different filters. A lower face velocity was chosen in this paper because the filter we prepared may be used as a mask $(5.34 \mathrm{~cm} / \mathrm{s})$. The dates in Figure 7 show that the filtration efficiency of cotton gauze differs enormously from nanofiber web for removing smaller particles (particle size lower than $0.7 \mu \mathrm{m}$ ), a clear U glyph filtration efficiency curve of cotton gauze is observed, the filtration efficiency of cotton gauze is lower than $80 \%$ when the particle size is smaller than $0.7 \mu \mathrm{m}$, and the particles about $0.4 \mu \mathrm{m}$ are the most difficult to filter out for common filter such as gauze mask (filtration efficiency: 36.1\%); however, the filtration efficiency of $\mathrm{Ag} / \mathrm{HBP} / \mathrm{PAN}$ nanofiber web easily reached $99.9 \%$. The excellent filtration efficiency of nanofiber web is mainly due to the ultrafine fiber (about $180 \mathrm{~nm}$ ), small pore diameter (less than $1 \mu \mathrm{m})$, high porosity $(86.4 \%)$, and BET $\left(25.3 \mathrm{~m}^{2} / \mathrm{g}\right)$ (shown in Table 2). The thickness of nanofiber web was 
TABLE 2: Characterization of nanofiber web.

\begin{tabular}{lccccc}
\hline Samples & Spinning time $(\mathrm{min})$ & Thickness $(\mu \mathrm{m})$ & Fiber diameter $(\mathrm{nm})$ & BET $\left(\mathrm{m}^{2} / \mathrm{g}\right)$ & Porosity $(\%)$ \\
\hline PAN & 20 & 88.5 & $176.4 \pm 42.5$ & 26.7 & 85.9 \\
Ag/HBP/PAN-1 & 20 & 81.2 & $188.1 \pm 31.6$ & 25.3 & 86.4 \\
Ag/HBP/PAN-2 & 40 & 153.9 & $181.7 \pm 26.9$ & 24.4 & 84.8 \\
Ag/HBP/PAN-3 & 60 & 230.6 & $184.4 \pm 30.3$ & 25.7 & 85.5 \\
\hline
\end{tabular}

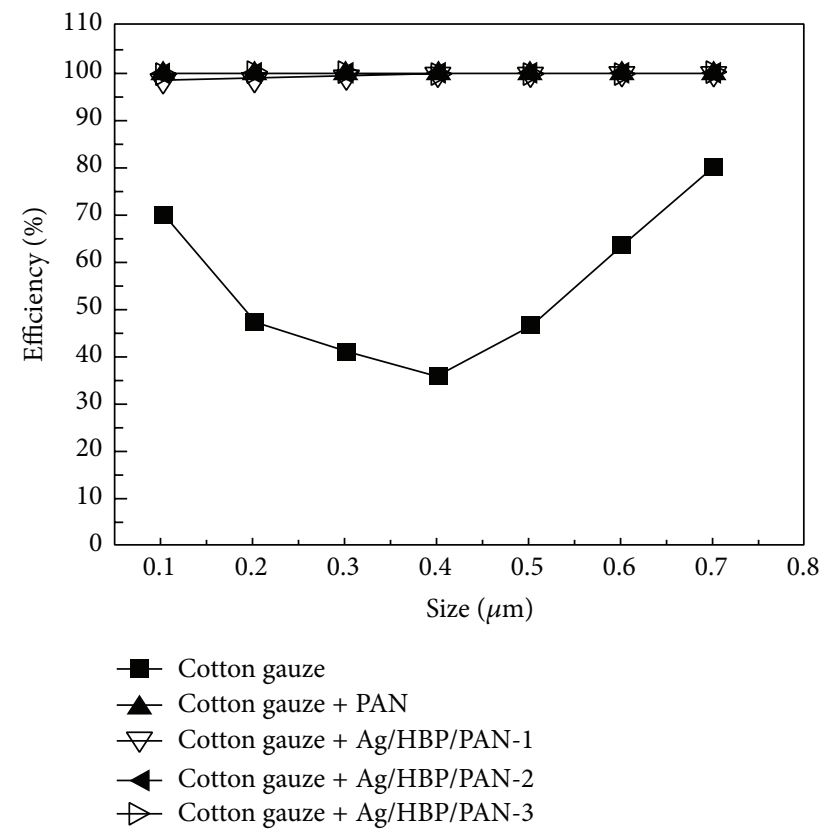

FIGURE 7: Filtration efficiency of filters.

controlled by spinning time in our experiment, which almost has no effects on its filtration efficiency.

The pressure drop of the filters is shown in Figure 8. Clearly, higher pressure drop is observed for $\mathrm{Ag} / \mathrm{HBP} / \mathrm{PAN}$ nanofiber web. We know that small pore diameter, high porosity, and thickness will improve the filtration efficiency of filters. However, the factors mentioned above are also the key reasons for increasing the pressure drop. Therefore, smaller thickness of nanofiber web is necessary for decreasing the pressure drop when its mechanical property is enough during use. The pressure drop of $\mathrm{Ag} / \mathrm{HBP} / \mathrm{PAN}$ nanofiber web is a little higher than the PAN nanofiber web, which may be attributed to the pore change in nanofiber web during the process of Ag-containing treatment.

\section{Conclusions}

The amino-terminated hyperbranched polymer (HBP) was synthesized in this paper at first; then the HBP was used as reductant and stabilizer to prepare $\mathrm{Ag} / \mathrm{HBP}$ aqueous solution. The silver nanoparticles were prepared with a mean diameter of about $10 \mathrm{~nm}$ and they have an outstanding disperse ability and stability in aqueous solution. The uniform PAN nanofiber web was prepared by electrospinning and treated with Ag/HBP solution under water bath. The SEM

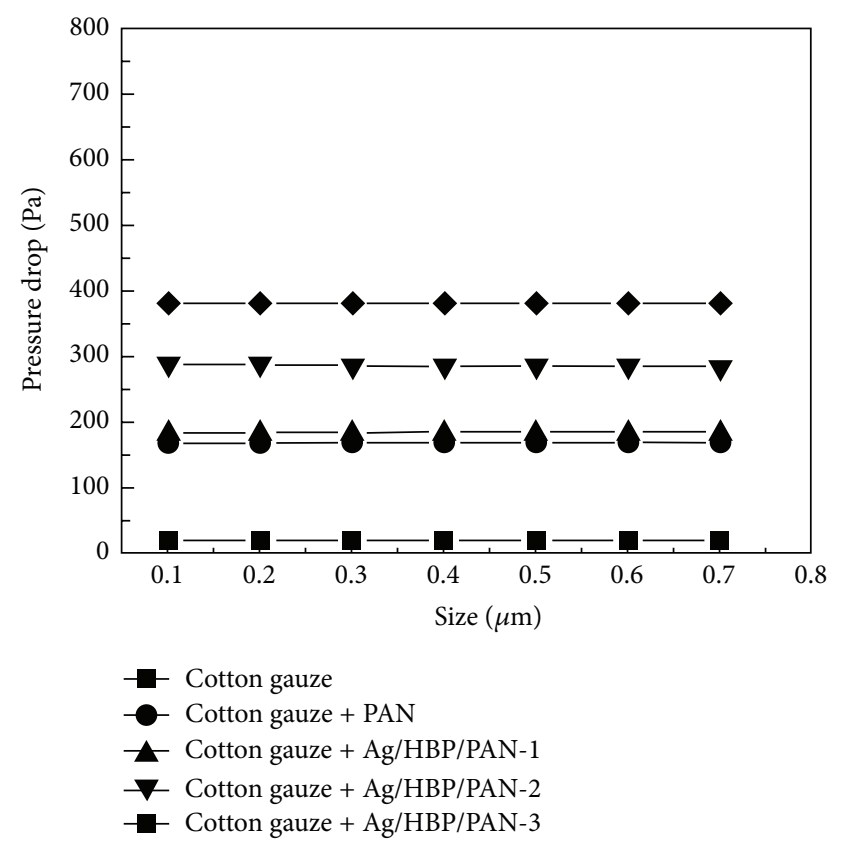

FIGURE 8: Pressure drop of filters.

and EDS showed that it was difficult for silver nanoparticles to be fixed on the surface of PAN nanofiber under low temperature $\left(30^{\circ} \mathrm{C}\right)$ and the content of silver nanoparticles on PAN nanofiber increased from $2.45 \mathrm{wt}$ \% to $10.84 \mathrm{wt} . \%$ when temperature rose to $90^{\circ} \mathrm{C}$. The antibacterial activity and the bacterial reduction rates of $S$. aureus and $E$. coli of HBP-treated PAN nanofiber are about 89\%; however, the Ag/HBP/PAN nanofiber showed better antibacterial property and it had $99.9 \%$ bacterial reduction of Staphylococcus aureus and $99.96 \%$ bacterial reduction of Escherichia coli, respectively, which may be due to the cooperative effects from the amino groups in HBP and Ag nanoparticles. The filtration efficiency of cotton gauze differs enormously from nanofiber web for removing smaller particles (particle size lower than $0.7 \mu \mathrm{m}$ ), a clear U glyph filtration efficiency curve of cotton gauze was observed, and the filtration efficiency of gauze mask (14 layers) was lower than $80 \%$ and as low as $36.1 \%$ for the particles of $0.4 \mu \mathrm{m}$; however, the filtration efficiency of PAN nanofiber web easily reached $99.9 \%$. Compared to lower pressure drop of gauze mask (about $20 \mathrm{~Pa}$ ), a further cut in pressure drop of Ag-containing nanofiber web is the primary mission in our future work.

\section{Competing Interests}

The authors declare that there are no competing interests. 


\section{Acknowledgments}

This work was supported by Liaoning Provincial Key Laboratory of Functional Textile Materials, National Natural Science Fund (51503105 and 51343002), College Students Innovation and Entrepreneurship Training Program in Jiangsu Province (201410304028Z), and Liaoning BaiQianWan Talents Program.

\section{References}

[1] S. M. S. Shahabadi, A. Kheradmand, V. Montazeri, and H. Ziaee, "Effects of process and ambient parameters on diameter and morphology of electrospun polyacrylonitrile nanofibers," Polymer Science Series A, vol. 57, no. 2, pp. 155-167, 2015.

[2] M. Yanilmaz and X.-W. Zhang, "Polymethylmethacrylate/polyacrylonitrile membranes via centrifugal spinning as separator in Li-ion batteries," Polymers, vol. 7, no. 4, pp. 629-643, 2015.

[3] M. A. Al-Omair, "Synthesis of antibacterial silver-poly( $\varepsilon$-caprolactone)-methacrylic acid graft copolymer nanofibers and their evaluation as potential wound dressing," Polymers, vol. 7, no. 8, pp. 1464-1475, 2015.

[4] J.-J. Xu, B.-H. Xu, D. Shou, X.-J. Xia, and Y. Hu, "Preparation and evaluation of vancomycin-loaded $\mathrm{N}$-trimethyl chitosan nanoparticles," Polymers, vol. 7, no. 9, pp. 1850-1870, 2015.

[5] V. Mottaghitalab and A. K. Haghi, "A study on electrospinning of polyacrylonitrile nanofibers," Korean Journal of Chemical Engineering, vol. 28, no. 1, pp. 114-118, 2011.

[6] D.-G. Yu, X.-Y. Li, X. Wang, J.-H. Yang, S. W. Annie Bligh, and G. R. Williams, "Nanofibers fabricated using triaxial electrospinning as zero order drug delivery systems," ACS Applied Materials \& Interfaces, vol. 7, no. 33, pp. 18891-18897, 2015.

[7] D.-G. Yu, C. Yang, M. Jin et al., "Medicated Janus fibers fabricated using a Teflon-coated side-by-side spinneret," Colloids and Surfaces B: Biointerfaces, vol. 138, pp. 110-116, 2016.

[8] D.-G. Yu, K. White, N. Chatterton, Y. Li, L. Li, and X. Wang, "Structural lipid nanoparticles self-assembled from electrospun core-shell polymeric nanocomposites," RSC Advances, vol. 5, no. 13, pp. 9462-9466, 2015.

[9] I. Heidari, M. M. Mashhadi, and G. Faraji, "A novel approach for preparation of aligned electrospun polyacrylonitrile nanofibers," Chemical Physics Letters, vol. 590, pp. 231-234, 2013.

[10] L. W. Ji, A. J. Medford, and X.-W. Zhang, "Effects of process and ambient parameters on diameter and morphology of electrospun polyacrylonitrile nanofibers," Polymer, vol. 50, no. 2, pp. 605-612, 2009.

[11] R. Nirmala, K. Jeon, R. Navamathavan, B.-S. Kim, M.-S. Khil, and H. Y. Kim, "Fabrication and characterization of II-VI semiconductor nanoparticles decorated electrospun polyacrylonitrile nanofibers," Journal of Colloid and Interface Science, vol. 397, pp. 65-72, 2013.

[12] Y. Tong, X.-F. Lu, W.-N. Sun, G. Nie, L. Yang, and C. Wang, "Electrospun polyacrylonitrile nanofibers supported $\mathrm{Ag} / \mathrm{Pd}$ nanoparticles for hydrogen generation from the hydrolysis of ammonia borane," Journal of Power Sources, vol. 261, pp. 221226, 2014.

[13] K. Yoon, K. Kim, X. Wang, D. Fang, B. S. Hsiao, and B. Chu, "High flux ultrafiltration membranes based on electrospun nanofibrous PAN scaffolds and chitosan coating," Polymer, vol. 47, no. 7, pp. 2434-2441, 2006.
[14] Y.-Z. Shi, Y.-J. Li, J.-F. Zhang, Z. Yu, and D. Yang, "Electrospun polyacrylonitrile nanofibers loaded with silver nanoparticles by silver mirror reaction," Materials Science and Engineering $C$, vol. 51, pp. 346-355, 2015.

[15] C. Q. Zhang, Q. B. Yang, N. Q. Zhan et al., "Silver nanoparticles grown on the surface of PAN nanofiber: preparation, characterization and catalytic performance," Colloids and Surfaces A: Physicochemical and Engineering Aspects, vol. 362, no. 1-3, pp. 58-64, 2010.

[16] D.-Y. Lee, K.-H. Lee, B.-Y. Kim, and N.-I. Cho, "Silver nanoparticles dispersed in electrospun polyacrylonitrile nanofibers via chemical reduction," Journal of Sol-Gel Science and Technology, vol. 54, no. 1, pp. 63-68, 2010.

[17] J. Bai, Q. B. Yang, S. Wang, and Y. Li, "Preparation and characterization of electrospun Ag/polyacrylonitrile composite nanofibers," Korean Journal of Chemical Engineering, vol. 28, no. 8, pp. 1761-1763, 2011.

[18] M. A. Kudryashov, A. I. Mashin, A. S. Tyurin, A. E. Fedosov, G. Chidichimo, and G. de Filpo, "Morphology of a silver/polyacrylonitrile nanocomposite," Technical Physics, vol. 56, no. 1, pp. 92-96, 2011.

[19] G. Panthi, S.-J. Park, T. W. Kim et al., "Electrospun composite nanofibers of polyacrylonitrile and $\mathrm{Ag}_{2} \mathrm{CO}_{3}$ nanoparticles for visible light photocatalysis and antibacterial applications," Journal of Materials Science, vol. 50, no. 13, pp. 4477-4485, 2015.

[20] Q. Shi, N. Vitchuli, J. Nowak et al., "Durable antibacterial $\mathrm{Ag} /$ polyacrylonitrile (Ag/PAN) hybrid nanofibers prepared by atmospheric plasma treatment and electrospinning," European Polymer Journal, vol. 47, no. 7, pp. 1402-1409, 2011.

[21] A. Mahapatra, N. Garg, B. P. Nayak, B. G. Mishra, and G. Hota, "Studies on the synthesis of electrospun PAN-Ag composite nanofibers for antibacterial application," Journal of Applied Polymer Science, vol. 124, no. 2, pp. 1178-1185, 2012.

[22] D.-G. Yu, C. J. Branford-White, N. P. Chatterton et al., "Electrospinning of concentrated polymer solutions," Macromolecules, vol. 43, no. 24, pp. 10743-10746, 2010.

[23] A. Tyurin, G. de Filpo, D. Cupelli, F. P. Nicoletta, A. Mashin, and G. Chidichimo, "Particle size tuning in silver-polyacrylonitrile nanocomposites," Express Polymer Letters, vol. 4, no. 2, pp. 7178, 2010.

[24] I. Karbownik, O. Rac, M. Fiedot, P. Suchorska-Woźniak, and H. Teterycz, "In situ preparation of silver-polyacrylonitrile nanocomposite fibres," European Polymer Journal, vol. 69, pp. 385395,2015

[25] M. A. Kudryashov, A. I. Mashin, A. S. Tyurin, G. Chidichimo, and G. De Filpo, "Metal-polymer composite films based on polyacrylonitrile and silver nanoparticles preparation and properties," Journal of Surface Investigation, vol. 4, no. 3, pp. 437441,2010

[26] H. Dodiuk-Kenig, K. Lizenboim, S. Roth et al., "Performance enhancement of dental composites using electrospun nanofibers," Journal of Nanomaterials, vol. 2008, Article ID 840254, 6 pages, 2008.

[27] W.-W. Zhang, D.-S. Zhang, Y.-Y. Chen, and H. Lin, "Hyperbranched polymer functional $\mathrm{TiO}_{2}$ nanoparticles: synthesis and its application for the anti-UV finishing of silk fabric," Fibers and Polymers, vol. 16, no. 3, pp. 503-509, 2015.

[28] F. Zhang and L. R. Yao, "Preparation of nano-silver solution," China Patent, ZL 201010166358.3, 2011. 
[29] G. Zhang, Y. Liu, H. Morikawa, and Y. Chen, "Application of $\mathrm{ZnO}$ nanoparticles to enhance the antimicrobial activity and ultraviolet protective property of bamboo pulp fabric," Cellulose, vol. 20, no. 4, pp. 1877-1884, 2013.

[30] E. S. Abdel-Halim and S. S. Al-Deyab, "Utilization of hydroxypropyl cellulose for green and efficient synthesis of silver nanoparticles," Carbohydrate Polymers, vol. 86, no. 4, pp. 16151622, 2011. 

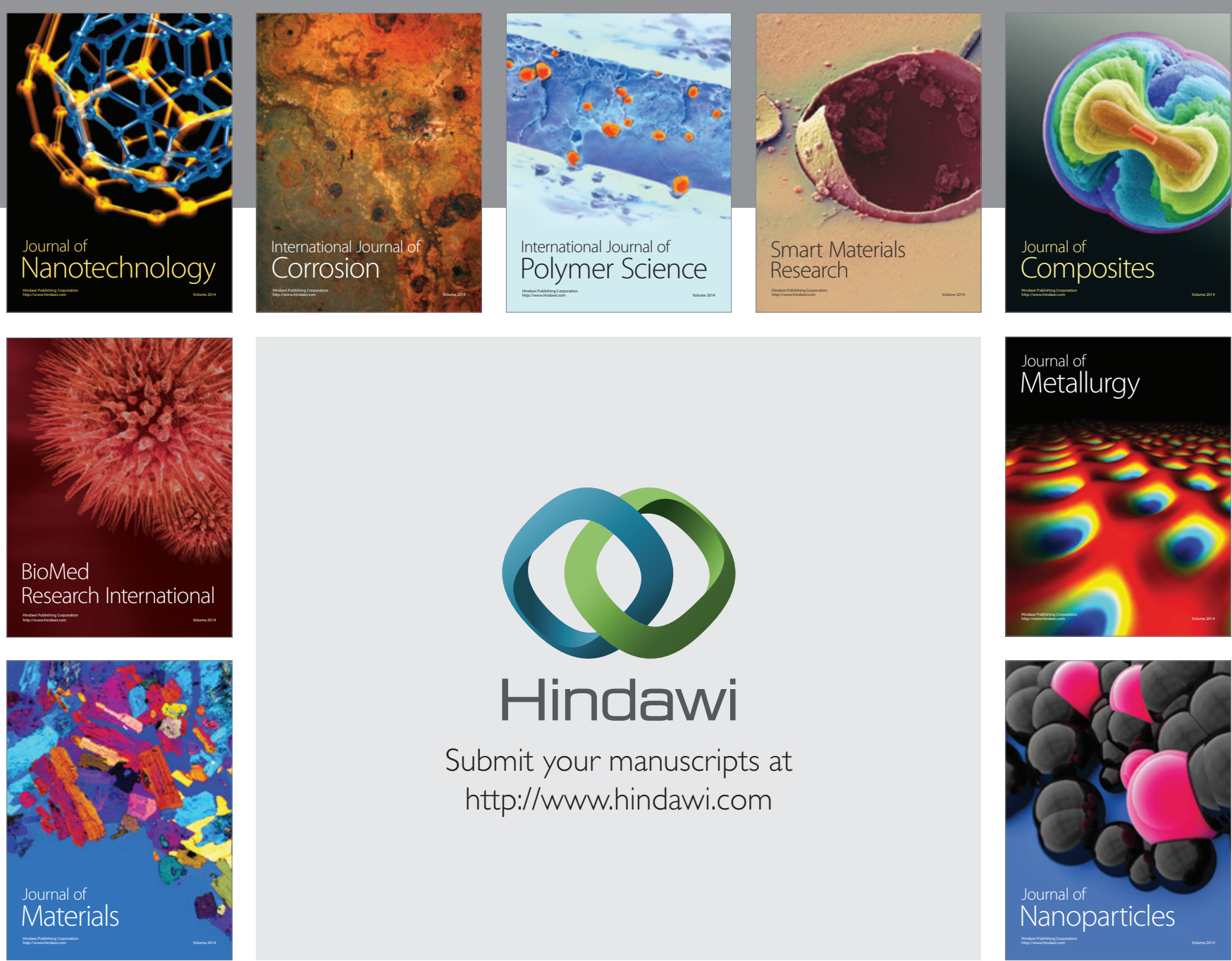

\section{Hindawi}

Submit your manuscripts at

http://www.hindawi.com

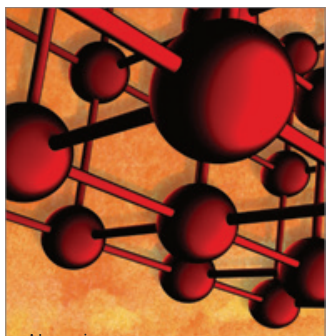

Materials Science and Engineering
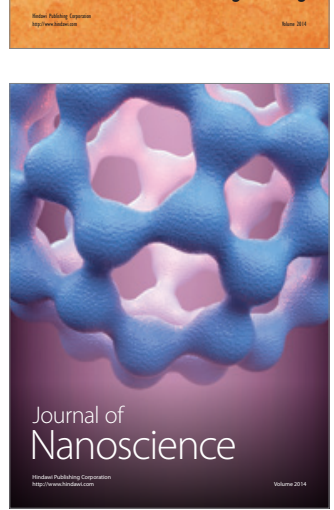
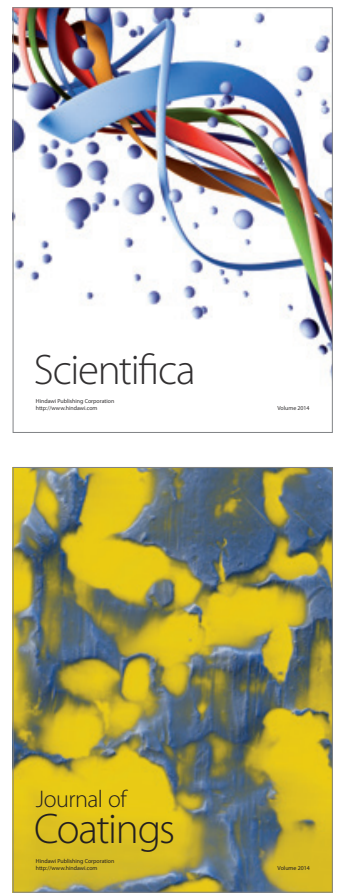
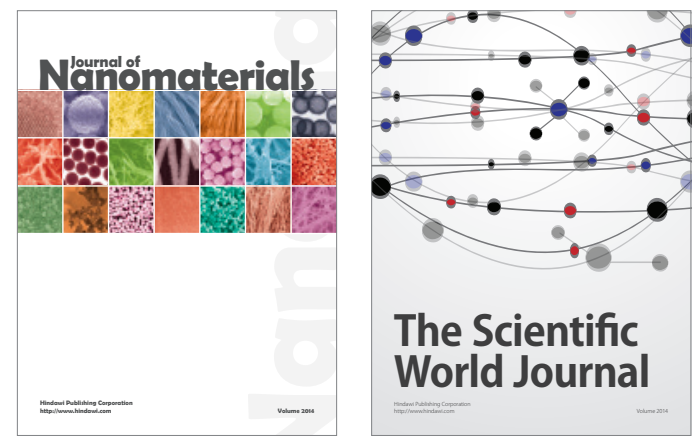

The Scientific World Journal
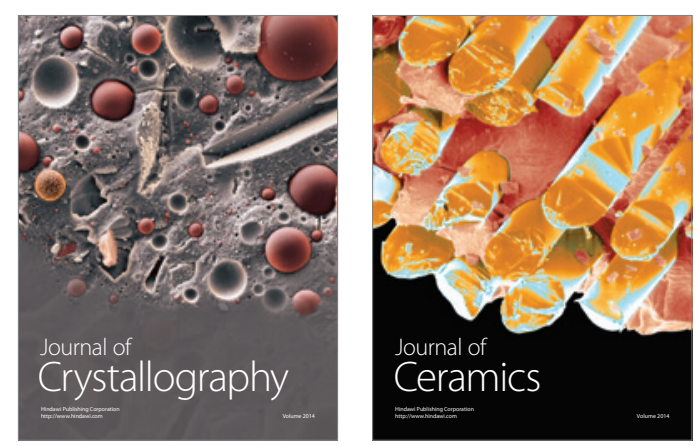
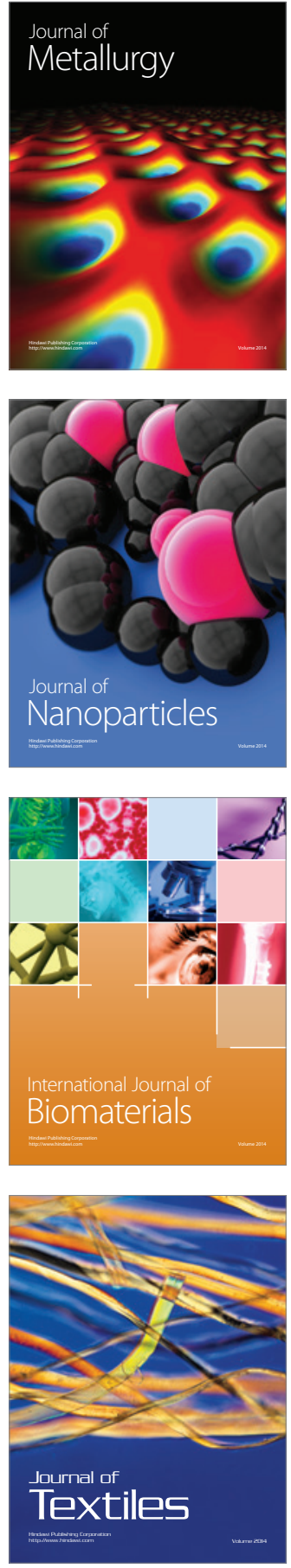